\title{
VACCINE-RELATED ENCEPHALOPATHY REDIAGNOSED AS DRAVET SYNDROME
}

Five patients with a history of seizures in the first year of life, occurring within 24 hours of DPT vaccination, were subsequently tested positive for $S C N I A$, and with support of genetic testing were diagnosed with Dravet syndrome, in a report from San Antonio Military Medical Center; Northwestern University School of Medicine, Children's Memorial Epilepsy Center, Chicago; Baylor College of Medicine, and Texas Children's Hospital, Houston, TX. Recurrent seizures in the first year of life were febrile and afebrile, generalized and unilateral, and often prolonged. After 1 year, seizures were often partial, myoclonic, and atypical absence. They were intractable to medication. Later, patients were often developmentally delayed, cognitively impaired and sometimes autistic. MRI was negative. The case report is intended to raise awareness of Dravet syndrome and aid in the early recognition and diagnosis. (Reyes IS, Hsieh DT, Laux LC, Wilfong AA. Alleged cases of vaccine encephalopathy rediagnosed years later as Dravet syndrome. Pediatrics Sept 2011;128:e699-e702). (Respond: David T Hsieh MD, Division of Child Neurology, San Antonio Military Medical Center, 3851 Roger Brooke Dr, Fort Sam Houston, TX 78234. E-mail: david.hsieh@us.af.mil).

COMMENT. Dravet syndrome is a rare genetic epileptic encephalopathy, recognized by ILAE as severe myoclonic epilepsy of infancy, and associated with mutations in SCNIA gene (neuronal sodium channel). Seizure onset is in the first year of life, and is often provoked by fevers. In the above cases, the close temporal relation of the pertussis vaccination to the first seizure may indicate a triggering effect (McIntosh AM et al. Lancet Neurol 2010;9(6):592-598). Fever alone is an unlikely precipitating factor, since the first seizure was afebrile in 2 of the 5 patients. Patients with seizures that have a close temporal relation to vaccination and are followed by frequent recurrences should be tested for SCN1A mutation. Mutations in the SCN1A gene are found in up to $80 \%$ of cases of Dravet syndrome. (Korff C et al. J Child Neurol 2007;22(2):185-194).

\section{METABOLIC DISORDERS}

\section{MRI FINDINGS AND OUTCOME IN MOLYBDENUM COFACTOR DEFICIENCY}

Researchers at Great Ormond Street Hospital, London, UK examined the clinical, brain MRI, biochemical, genetic, and EEG features and outcome in 8 children with a diagnosis of molybdenum cofactor deficiency seen over a 10-year period. Six neonates had an early (classical) onset with predominantly epileptic encephalopathy and neonatal seizures, and 2 had a late (atypical) onset with global developmental impairment. Four were boys. Neonatal history was normal except in one infant with presumed HIE who required ventilatory support for 3 days. Age at presentation ranged from 1 day to 24 months. Clinical features leading to diagnosis included progressive microcephaly, dysmorphisms, progressive pyramidal and extrapyramidal signs, axial hypotonia, kyphoscoliosis, verbal dyspraxia, and visual impairment due to cortical damage or lens 
subluxation. Follow-up ranged from 12 months to 8 years. No patient died. Time to biochemical diagnosis (high urine sulfite and low plasma urate, high urine purine metabolites [xanthines] and 5-sulfocysteine) ranged from 4 days to 4 years (median 3 months). EEG abnormalities included burst suppression pattern and seizure activity. Brain MRI showed cerebral infarction in all except one with atypical onset. Distinctive features seen in an early brain MRI were acute infarction of the globus pallidi and subthalamic regions with older cerebral hemisphere infarction, chronic lesions suggestive of a prenatal insult, pontocerebellar hypoplasia and retrocerebellar cyst, and a band at the cortical/subcortical white matter. Sequential imaging showed progressive pontine atrophy and enlargement of retrocerebellar cyst. Early brain MRI (<1 week) abnormality may lead to early diagnosis and treatment. (Vijayakumar K, Gunny R, Grunewald S, et al. Clinical neuroimaging features and outcome in molybdenum cofactor deficiency. Pediatr Neurol October 2011;45;246-252). (Respond: Dr Prabhakar, Department of Neurology, Great Ormond Street Hospital, London WC1N 3JH, UK. E-mail: prabhp@gosh.nhs.uk).

COMMENT. Molybdednum cofactor deficiency is a rare autosomal recessive neurodegenerative disorder characterized by deficiency of molybdenum-dependent enzymes xanthine oxidase, sulfite oxidase, nitrogenases, and nitrate reductase. Presentation includes intractable seizures, severe global delay, feeding difficulties, followed by frequent infant fatalities. Milder phenotypes are described with late presentation and more sanguine prognosis. The above description of a specific MRI pattern may lead to early prenatal diagnosis, mutational confirmation and therapeutic intervention.

\section{CO-ENZYME Q10 AND L-CARNITINE IN CYCLIC VOMITING}

Researchers at Childrens Hospital Los Angeles, CA conducted a retrospective chart review of 42 patients treated with co-enzyme Q10 and L-carnitine, and the addition of amitryptiline (or cyproheptadine for $<5$ year olds) in refractory cases. Patients ate 3 meals and 3 snacks a day and avoided fasting. Treatment was monitored with blood levels: co-enzyme Q10 >3.0 mg/L; L-carnitine >40 micromolar; amitryptiline >150 $\mathrm{ng} / \mathrm{ml}$; cyproheptadine dose $0.5 \mathrm{mg} / \mathrm{kg} /$ day. In 30 cases with available outcome data, vomiting episodes resolved in 23 , and improved by $>50-75 \%$ in 4 cases. Treatment with. mitochondrial-targeted cofactors (co-enzyme Q10 and L-carnitine) plus amitryptiline was highly effective in the prevention of vomiting episodes. (Boles RG. High degree of efficacy in the treatment of cyclic vomiting syndrome with combined co-enzyme Q10, Lcarnitine and amitryptiline, a case series. BMC Neurology 2011;11:102-107). (Respond: E-mail: rboles@chla.usc.edu).

COMMENT. The author observes that these findings need confirmation by a prospective blinded trial in unselected cases. Cyclic vomiting is usually regarded as a form of migraine, and mitochondrial dysfunction may be a factor in etiology of both cyclic vomiting and migraine. Cyclic vomiting, sometimes associated with epilepsy and epileptiform EEG, may also respond to treatment with antiepileptic agents (topiramate, valproate)(Olmez A et al. Pediatr Neurol 2006;35(5):348-351)(Millichap JG, Lombroso CT, Lennox WG. Pediatrics 1955;15(6):705-714). 